\title{
EVALUATION OF EFFICACY OF PLATELET-RICH FIBRIN (PRF) VERSUS ALVOGYL AND ZINC OXIDE AND EUGENOL (ZOE) PACKING IN THE MANAGEMENT OF ALVEOLAR OSTEITIS: A PROSPECTIVE RANDOMIZED CLINICAL STUDY
}

\author{
Sayed A. Rashed*, Ahmed T. Elsharkawy ${ }^{* *}$ and Amira Zaied ${ }^{* * *}$
}

\begin{abstract}
Purpose: The purpose of this research was to compare the effectiveness of platelet rich fibrin (PRF), alvogyl and zinc oxide/ eugenol (ZOE) intra-alveolar dressings for pain relief and socket healing (Epithelialization) in dry socket management and to study relevant epidemiological features.

Patients and methods: A total of 45 patients with alveolar osteitis were randomly divided into three groups; Group (A) patients received PRF, Group (B) patients received Alvogyl and Group (C) patients received ZOE dressing. All the patients were evaluated for Pain (VAS), degree of inflammation, healthy granulation tissue formation and number of exposed socket walls (socket epithelialization) at $1^{\text {st }}, 3^{\text {rd }}, 7^{\text {th }}$, and $14^{\text {th }}$ post-operative day.
\end{abstract}

Results: Group A (PRF) showed better and faster socket healing than Group B and C. However, symptomatic pain relief was faster in Group B (Alvogyl) than in Group A and C.

Conclusion: PRF in this study illustrates the promising results to be used effectively as the suitable dressing material in the management of alveolar osteitis. PRF treated cases showed a shorter time required for complete and fast clinical healing.

KEY WORDS: alveolar Osteitis, Dry socket, (PRF), Alvogyl, zinc oxide/ eugenol (ZOE)

\section{INTRODUCTION}

Acute alveolar osteitis (AO) is one of the most common postoperative complications following extraction of permanent teeth. It was defined by Blum ${ }^{1}$ as "The presence of postoperative pain in and around the site of extraction which increases in severity between 1 and 3 days after the extraction, accompanied by a partially or totally disintegrated blood clot within the alveolar socket, with or without halitosis". Clinical features of the dry socket include severe throbbing pain with an extraction socket devoid of clot and/or exposed bone and edema of

\footnotetext{
* Lecturer, Department of Oral and Maxillofacial Surgery, Faculty of Dentistry, Ahram Canadian University.

** Lecturer, Department of Oral and Maxillofacial Surgery, Faculty of Dentistry, Cairo University.

*** Lecturer, Department of Oral and Maxillofacial Surgery, Faculty of Dentistry, Fayoum University
} 
surrounding gingiva with or without associated regional lymphadenitis. The phrase "dry socket" was first formulated by Crawford ${ }^{2}$ in 1896, but there are many different other names as alveolitis sicca, sicca dolorosa, alveolar osteitis, fibrinolytic osteitis, septic socket; however the term dry socket had popularity. ${ }^{3-5}$

Alveolar osteitis AO can affect both jaws with greater incidence on the lower jaw. AO occurs mainly after extraction of impacted mandibular third molars. AO affects women up to five times more than men and is more common in ongoing smokers. The etiology of AO is not fully clarified, but the fibrinolysis of the the blood clot as a result of bacterial invasion is the most common cause. It has been hypothesized that trauma during extraction or the presence of a bacterial infection hastens the release of plasminogen tissue activators resulting in the plasmin induction of fibrinolysis that disintegrates formed blood and causing a dry socket. It has been believed that dry socket has a multifactorial etiology, which can be divided into general and local factors such as: traumatic extraction, smoking, intraligamentary anesthetic injection contraceptive use, etc. Several studies suggest a direct correlation between contraceptive use and dry socket. Contraceptives contain estrogen, which is believed to affect the coagulation system. ${ }^{6-8}$

Alveolar osteitis AO requires extra time for its treatment, making it a socially significant disorder. Systemic antibiotics, topical antibiotics, chlorhexidine, tranexemic acid, steroids, etc.; have been proposed to assist in the prevention of AO. Recently, good results have been reported, with using PRF, for prevention of dry socket after removing lower third molars. PRF will act as a stable blood clot for neovascularization and accelerated tissue regeneration. This can be used to improve wound healing. ${ }^{9-13}$

The primary aim of AO management is pain control until initiation of normal healing, and in the vast majority, local measures alone are satisfactory but in some cases, systemic analgesics or antibiotics may be necessary. Treatment of AO includes modifications in the surgical technique, use of antibiotics, mouth rinsing with antimicrobial agents, placement of different medications in the socket, etc. The use of intra-alveolar dressing materials such as zinc oxide and eugenol ZNE dressing and alvogyl packing is widely suggested in the literature, although it is generally accepted that some dressings delay healing of the extraction socket. Despite all the researches, alveolar osteitis is still a challenge in terms of delayed healing and frequent visits by the patients. ${ }^{14-17}$

The use of platelet concentrates for the improvement of soft and hard tissues regeneration is one of the latest achievements in dentistry. These platelet concentrates are obtained by centrifugation of fresh venous blood drawn from the patient's vein. Platelet-rich plasma (PRP) is a first generation concentrate; the blood is collected with an anticoagulant. It is essential to add chemicals, such as calcium chloride and bovine thrombin to make a gel. But later it has been reported that PRP has a low effect on tissue regeneration. Platelet-rich fibrin (PRF) a second- generation aggregate was developed in 2001 in France by Prof. Dr. Joseph Choukroun ${ }^{18}$. PRF is an autologous fibrin matrix rich in platelets, leucocytes and monocytes that induce a cascade of multiple growth factors release. It is a simple technique, the blood is collected without any anticoagulant, immediately centrifuged and the PRF protocol doesn't need biochemical additives to make a gel. The PRF clot is achieved through a natural polymerization process during centrifugation and its natural and stable fibrin matrix is responsible for tissue regeneration. ${ }^{19-22}$

So, the present study was undertaken to evaluate the effectiveness of PRF in the management of alveolar osteitis and to compare the results to the traditional zinc oxide eugenol dressing and alvogyl packing. 


\section{PATIENTS AND METHODS}

Forty five patients (29 female and 16 male) with $\mathrm{AO}$ were included in the study. The mandible was affected in 28 of the cases and 17 cases in the maxilla. All patients were diagnosed and treated in the Department of Oral and maxillofacial surgery, Dental College, Ahram Canadian University. Informed consent was obtained from all the patients. After obtaining approval from the ACU research and ethical committee, patients were randomly divided into three groups: Group A: patients received PRF, Group B received Alvogyl (Manufactured by Septodont, France. Content: iodoform, eugenol and butamben) and Group $C$ received as an obtundant dressing ZOE (Manufactured by DENTSPLY, USA)

\section{Inclusion criteria}

Diagnosis of AO was clinically established on the basis of: 1. Pain in and around the extraction socket that increases in severity from $1^{\text {st }}$ and $3^{\text {rd }}$ day after extraction. 2. Partial or total clot loss and any other associated findings such as halitosis, erythema, exposed socket walls and lymphadenopathy.

\section{Exclusion criteria}

Exclusion criteria included conditions such as pregnancy, history of radiotherapy, immunocompromised disorders, smokers, diabetics and patients on steroid therapy.

\section{Surgical technique}

After adequate anesthesia was established, the extraction wound was mechanically debrided and rinsed with normal saline (sodium chloride $0.9 \%$ ) (Egypt Otsuka Pharmaceuticals Co) to remove remnants of disintegrated blood clot and debris. The marginal gingiva around the socket was refreshed using a scalpel. The socket was treated with sterile gauze and was then filled with intraalveolar dressings. The edges of the wound were then sutured. Post-operative instructions were given and patients were recalled for follow up on $1^{\text {st }}, 3^{\text {rd }}$, $5^{\text {th }}, 7^{\text {th }}, 14^{\text {th }}$ and subsequent post-operative days until healing occurred (Fig 1, 2, 3).

\section{Method of preparation of platelet-rich fibrin}

PRF preparation was performed according to Dohan ${ }^{19}$. The procedure was explained to the patients. Also, the patients were informed about the complications or accidents of phlebotomy, hematoma, edema, syncope or the doctor can't find the vein. The area of the anticubital region was prepared with alcohol wipes and $10 \mathrm{ml}$ of blood for every two tubes was drawn from the patients' cephalic or basilic veins using a safety blood collection set+ Luer adapter (Greiner BioOne GmbH, Austria) (Fig. 1B). The blood was then transferred to a centrifugal vial without any anticoagulant and centrifuged immediately using a tabletop centrifuge for 10 minutes at $2700 \mathrm{rpm}$. The resultant product consists of three layers :(1) Topmost layer consisting of acellular plasma (platelet poor plasma) (2) PRF clot in the middle which it is used clinically and (3) Red Blood Cells at the bottom that are discarded (Fig. 1C).

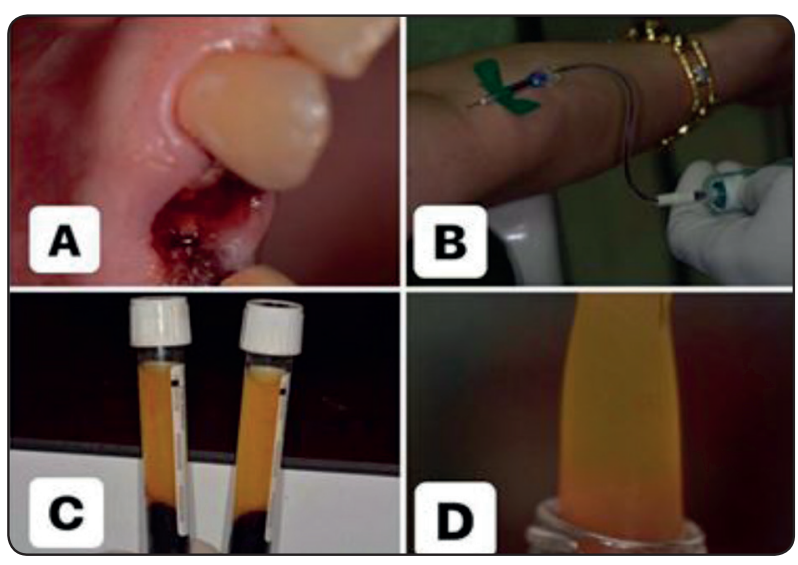

Fig. (1) A. Dry socket at upper first premolar B. Venous blood collection C. centrifugation vial with PRF in middle zone D. PRF plug

\section{Method of Placing PRF}

PRF clot was withdrawn from the centrifugal vial using Adson forceps. Most of the attached red blood cell was removed from the bottom of the PRF 


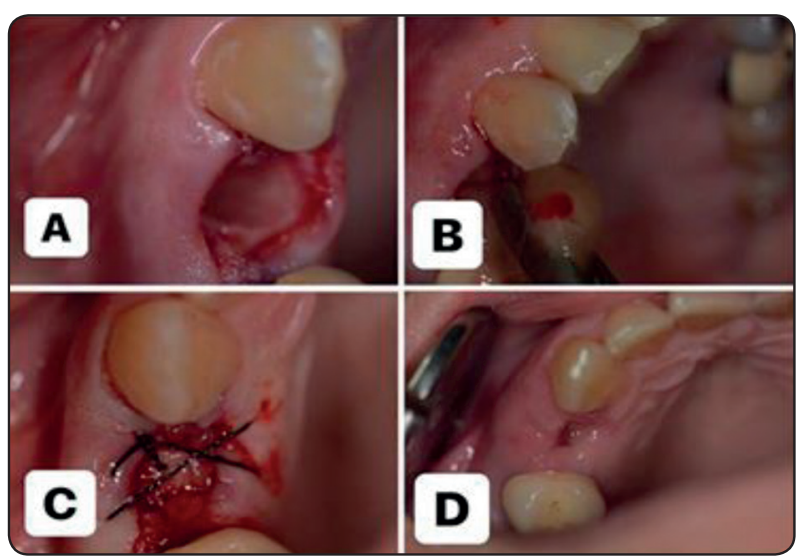

Fig. (2) A. Clean socket after debridement B. PRF placement C. Suturing to stabilize PRF D. One week postoperative healing of dry socket

using scissors, and then the PRF plug was condensed using special PRF processing box (Nichrmnox Co) to squeeze serum out of the Platelet-rich fibrin clot as described by Dohan ${ }^{19,20}$. The PRF plug was then placed in the cleaned socket and stabilized using figure of 8 black silk suture (Fig 2).

\section{Method of Placing Alvogyl}

A few fibers of Alvogyl was placed deep in the cleaned socket using a sterile dental tweezers ensuring that the exposed bone was completely covered, followed by the placement of sterile gauze which was removed after few minutes (Fig 3A, B and C).

\section{Method of Placing Zinc Oxide Eugenol ZOE}

A gauze piece soaked with ZOE paste was placed in the cleaned socket under aseptic technique. The pack was lightly inserted into the socket and patients were instructed not to eat for the next one hour (Fig $3 \mathrm{D}, \mathrm{E}$ and $\mathrm{F}$ ).

\section{Clinical parameters and variables}

Pain: The subjective feeling of pain were assessed on the basis of 10 point Visual Analogue Scale VAS, with a score of "0" equals "no pain" and " 10 "

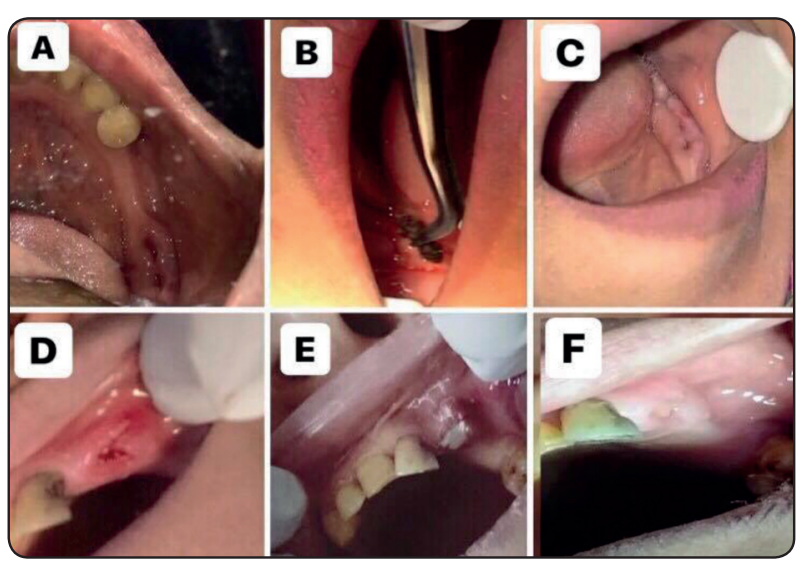

Fig. (3) A. Dry socket in lower molar area. B. Placement of Alvogyl. C. One week postoperative healing of dry socket. D. Dry socket of upper second premolar. E. ZNE dressing placement. F. Two weeks postoperative healing of dry socket.

equals "very severe pain" which was completed by the patient. The pain was evaluated pre-operatively on day 0 and post-operatively at day 1, 3, 5, and 7 , after placement of the curative dressing. The dressings were evaluated at every follow-up visit and were changed in case of persistence of pain. No further dressings were done if the patients had sustained pain relief. All the patients were asked not to take any pain killers in order to assess the antinociceptive property.

\section{Socket healing and Granulation tissue} formation: Clinical examinations of the dry socket healing were performed on $1^{\text {st }}, 3^{\text {rd }}, 5^{\text {th }}, 7^{\text {th }}$, and $14^{\text {th }}$ day and assessed by the the number of exposed socket walls. Evaluation of soft tissue healing was based on the standard method. ${ }^{23}$ Alveolar osteitis treated with PRF, Alvogyl and ZOE was assessed clinically by the coverage of the exposed bone by soft granulation tissue formation and socket epithelialization which can be graded as: 0 - no bony walls exposed, 1 - only one bony wall exposed, 2 - two bony walls exposed, 3 - three bony walls exposed, and 4 - four bony walls exposed. The granulation tissue was divided into healthy (pink in color and does not bleed on probing) and unhealthy granulation tissue (dark red in color and often bleeds 
on probing). Criteria for dry socket assessment were based on Blum's method. ${ }^{1}$ The patients were followed up until signs and symptoms disappear.

The degree of inflammation: Inflammation was assessed clinically by gentle probing of the extraction socket to ensure presence or absence of gingival bleeding and recorded as no, mild, moderate, and severe bleeding.

\section{Data collection}

Readings for each parameter was recorded preoperatively (baseline reading) and postoperatively on $1^{\text {st }}, 3^{\text {rd }}, 5^{\text {th }}, 7^{\text {th }}$ and $14^{\text {th }}$ day and on subsequent alternate days until complete healing occurred and the mean of those parameters was compared to the baseline reading. Data from the three groups were collected, tabulated and statistically analyzed using a SPSS statistical package. The data were summarized as means and standard deviations for the continuous outcome (pain), ANOVA used to compare between the mean values of the three groups. The data were summarized frequency for qualitative data (Postoperative inflammation score and exposed socket wall score). Chi square test used to compare the mean values of the three groups. The level of significance was set at $5 \%$ for all statistical analyses and confidence interval (CI) at $95 \%$. The level of significance was concluded at $P<0.05$ and the level of high significance was concluded at $P$ $<0.001$.

\section{RESULTS}

Out of forty five patients with dry socket, twenty nine were females (64.4\%) and sixteen were males $(35.6 \%)$. Patient age ranged from 17 to 56 years (mean $=39.8$ years) and most of the patients were in the third decade. The onset of dry socket was considered at the time of onset of pain after extraction. The vast majority of cases 26 (57.77 $\%)$ ] had onset on the $3^{\text {rd }}$ day after extraction and 12 $(26.67 \%)$ cases, manifested on the $2^{\text {nd }}$ day, while
$7(15.56 \%)$ cases noted symptoms on the $4^{\text {th }}$ day. Higher incidence of dry socket was found in the mandible $(62.22 \%)$ as compared to the maxilla $(37.78 \%)$. The incidence of dry socket was detected to be higher in cases of surgical removal than in simple extraction (the ratio was 3.5:1).

\section{Pain score}

The baseline clinical examination prior to the initiation of treatment revealed severe pain in all patients.

Figure (4) shows that the three groups showed a statistically significant decrease in pain scores (VAS values) at $1^{\text {st }}, 3^{\text {rd }}, 5^{\text {th }}$ and $7^{\text {th }}$ days. There is statistically significantly less pain and better pain remission for Alvogyl group compared to other groups followed by $\mathbf{P R F}$ at $1^{\text {st }}, 3^{\text {rd }}, 5^{\text {th }}$ days However; the difference was non-significant on day 7 between three groups.

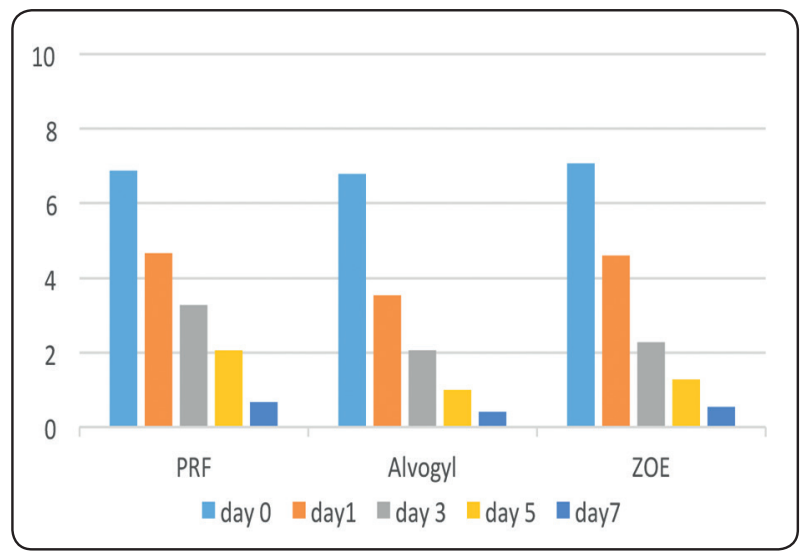

Fig (4): Comparison of pain scores index of patients in three groups

\section{Inflammation score:}

Figure (5) shows that the three groups showed highly statistically significant improvement in inflammation score at $1^{\text {st }}, 3^{\text {rd }}$, and $5^{\text {th }}$ day. PRF group showing more improvement with better reduction in inflammation compared to the other two groups followed by Alvogyl. However; the difference was non-significant on day 7 and 14 between three groups showing nearly similar results. 


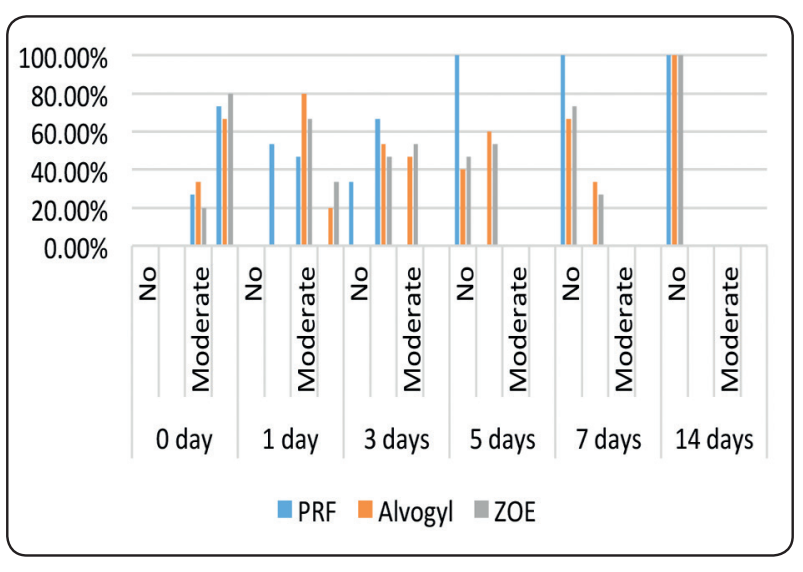

Fig (5): Comparison of inflammation score of patients in three groups

\section{Socket healing}

\section{Exposed socket wall score}

Table (1) shows that there was a statistically significant difference in the mean number of exposed socket wall scores between three groups at $3^{\text {rd }}$ and $7^{\text {th }}$ day with more improvements for PRF group as compared to three other groups. PRF Group showed less number of exposed socket walls and hence better healing than alvogyl and ZOE.

TABLE (1): Comparison of Exposed socket wall score of patients in three groups

\begin{tabular}{|c|c|c|c|c|c|c|c|c|}
\hline \multirow{2}{*}{\multicolumn{2}{|c|}{ Exposed Socket Walls }} & \multicolumn{2}{|c|}{ PRF } & \multicolumn{2}{|c|}{ Alvogyl } & \multicolumn{2}{|c|}{ ZOE } & \multirow{2}{*}{$P$ value } \\
\hline & & $\mathbf{N}$ & $\%$ & $\mathbf{N}$ & $\%$ & $\mathbf{N}$ & $\%$ & \\
\hline \multirow{5}{*}{ day 0} & 0 wall & 0 & $0.0 \%$ & 0 & $0.0 \%$ & 0 & $0.0 \%$ & \multirow{5}{*}{$0.896^{\#}$} \\
\hline & 1 wall & 2 & $13.3 \%$ & 2 & $13.3 \%$ & 4 & $26.7 \%$ & \\
\hline & 2 walls & 6 & $40.0 \%$ & 6 & $40.0 \%$ & 3 & $20.0 \%$ & \\
\hline & 3 walls & 4 & $26.7 \%$ & 5 & $33.3 \%$ & 5 & $33.3 \%$ & \\
\hline & 4 walls & 3 & $20.0 \%$ & 2 & $13.3 \%$ & 3 & $20.0 \%$ & \\
\hline \multirow{5}{*}{ day 1} & 0 wall & 0 & $0.0 \%$ & 0 & $0.0 \%$ & 0 & $0.0 \%$ & \multirow{5}{*}{$0.185^{\#}$} \\
\hline & 1 wall & 6 & $40.0 \%$ & 4 & $26.7 \%$ & 4 & $26.7 \%$ & \\
\hline & 2 walls & 9 & $60.0 \%$ & 8 & $53.3 \%$ & 6 & $40.0 \%$ & \\
\hline & 3 walls & 0 & $0.0 \%$ & 3 & $20.0 \%$ & 5 & $33.3 \%$ & \\
\hline & 4 walls & 0 & $0.0 \%$ & 0 & $0.0 \%$ & 0 & $0.0 \%$ & \\
\hline \multirow{5}{*}{ day 3} & 0 wall & 5 & $33.3 \%$ & 0 & $0.0 \%$ & 2 & $13.3 \%$ & \multirow{5}{*}{$0.011^{\#}$} \\
\hline & 1 wall & 10 & $66.7 \%$ & 9 & $60.0 \%$ & 8 & $53.3 \%$ & \\
\hline & 2 walls & 0 & $0.0 \%$ & 6 & $40.0 \%$ & 5 & $33.3 \%$ & \\
\hline & 3 walls & 0 & $0.0 \%$ & 0 & $0.0 \%$ & 0 & $0.0 \%$ & \\
\hline & 4 walls & 0 & $0.0 \%$ & 0 & $0.0 \%$ & 0 & $0.0 \%$ & \\
\hline \multirow{5}{*}{ day 5} & 0 wall & 10 & $66.7 \%$ & 7 & $46.7 \%$ & 4 & $26.7 \%$ & \multirow{5}{*}{$0.075^{\#}$} \\
\hline & 1 wall & 5 & $33.3 \%$ & 8 & $53.3 \%$ & 8 & $53.3 \%$ & \\
\hline & 2 walls & 0 & $0.0 \%$ & 0 & $0.0 \%$ & 3 & $20.0 \%$ & \\
\hline & 3 walls & 0 & $0.0 \%$ & 0 & $0.0 \%$ & 0 & $0.0 \%$ & \\
\hline & 4 walls & 0 & $0.0 \%$ & 0 & $0.0 \%$ & 0 & $0.0 \%$ & \\
\hline \multirow{5}{*}{ day 7} & 0 wall & 15 & $100.0 \%$ & 10 & $66.7 \%$ & 8 & $53.3 \%$ & \multirow{5}{*}{$0.008^{*}$} \\
\hline & 1 wall & 0 & $0.0 \%$ & 5 & $33.3 \%$ & 7 & $46.7 \%$ & \\
\hline & 2 walls & 0 & $0.0 \%$ & 0 & $0.0 \%$ & 0 & $0.0 \%$ & \\
\hline & 3 walls & 0 & $0.0 \%$ & 0 & $0.0 \%$ & 0 & $0.0 \%$ & \\
\hline & 4 walls & 0 & $0.0 \%$ & 0 & $0.0 \%$ & 0 & $0.0 \%$ & \\
\hline \multirow{5}{*}{ day 14} & 0 wall & 15 & $100.0 \%$ & 15 & $100.0 \%$ & 12 & $80.0 \%$ & \multirow{5}{*}{$0.096^{\#}$} \\
\hline & 1 wall & 0 & $0.0 \%$ & 0 & $0.0 \%$ & 3 & $20.0 \%$ & \\
\hline & 2 walls & 0 & $0.0 \%$ & 0 & $0.0 \%$ & 0 & $0.0 \%$ & \\
\hline & 3 walls & 0 & $0.0 \%$ & 0 & $0.0 \%$ & 0 & $0.0 \%$ & \\
\hline & 4 walls & 0 & $0.0 \%$ & 0 & $0.0 \%$ & 0 & $0.0 \%$ & \\
\hline P value & & & $0001 *$ & & $001 *$ & & $01 *$ & \\
\hline
\end{tabular}

${ }^{\#}$ Fisher exact test; was used as $20 \%$ of the cells or more have expected count less than 5 


\section{DISCUSSION}

Alveolar osteitis is a common drawback of exodontia and results in irritability of the patient. Intra-socket medications like $\mathrm{ZO} / \mathrm{E}$ and Alvogyl have been utilized for treatment of dry socket. Dohan et al. ${ }^{21}$ suggested that PRF can correct destructive reactions in the natural process of healing; Thus PRF has immune regulatory mechanism rather than inflammatory modulation. The present study is an attempt to assess the healing potential of PRF in the management of dry socket in comparison with alvogyl and $\mathrm{ZO} / \mathrm{E}$. The rationale for using PRF in our study was based on previous studies, which showed the potential of PRF in the process of soft and hard tissue healing. A comparative assessment is essential in order to make a rational choice of three medicaments. In the present study efficacy of medicaments was evaluated on the basis of capability to provide pain relief and clinical evidence of socket healing.

In the present study females were seen more commonly affected $(64.4 \%)$; the reason behind this high percentage may be due to oral contraceptives use and menstruation. The present study implies that $\mathrm{AO}$ is more in the mandible $(62.22 \%)$ than in maxilla; these findings are similar with the observations in the previous studies ${ }^{24-26}$ which show a higher incidence in the lower jaw. Increased bone density and insufficient blood supply with reduced capability for granulation tissue formation could be attributed to an increased number in the lower jaw.

Findings of the present study correlate well with Akinbami and Godspower ${ }^{27}$, and Parthasarathi et $\mathrm{al}^{3}$ who reported the highest incidence of dry socket in $3^{\text {rd }}$ decade and with surgical extractions. However, it might due to the large percentage of surgically extracted mandibular molars with the effect of surgical trauma. Mamoun ${ }^{6}$, Nusair and Younis ${ }^{26}$, Mudali and Mohamed ${ }^{28}$ have reported that the incidence of the dry socket was higher after surgical extraction than simple extractions. This also correlates with results of our research. This could be due to more liberation of direct tissue activators secondary to traumatic extractions.

The present study showed that severe pain was measured in the VAS scale pre-operatively. The VAS scores for pain improved gradually during the follow-up period and the intensity of pain decreased in three groups over a period of time. This decrease was significantly more in Alvogyl group followed by the PRF group (Fig 4). Better pain relief with Alvogyl packing can be attributed to Alvogyl contain eugenol, which has a sedative effect, in addition it contains butamben with its anesthetic effect. Reductions in pain level by the use of PRF might be due to PRF action as an immune regulator and may decrease the deleterious effects of inflammation as described by the Dohan et al. ${ }^{21}$ It has been suggested that the fibrin matrix results in angiogenesis, therefore it provides natural resurfacing of the dry socket wound, which results in the covering of the exposed nerve endings in the socket leading to a marked decrease of associated pain.

In our study alveolar socket healing was faster and better in PRF group as compared to ZOE and Alvogyl groups at the $3^{\text {rd }}$ and $7^{\text {th }}$ post-treatment days. Though the management of alveolar osteitis with PRF showed much better results with improved socket healing and reduced inflammation, effective pain control and a lesser number of patient visits, along with being more economical, compared to ZOE dressing and Alvogyl packing. Exposed bony walls in dry sockets showed better healing with early granulation tissue formation at day 3 and 7 and complete coverage of healthy granulation tissue on $2^{\text {nd }}$ week post-operatively, after being treated with PRF (Fig 6). This is attributing PRF membrane has a slow continuous release of growth factors for at least seven and up to 28 days, which means the PRF membrane activates its background for a significant period. Those growth factors encourage the mitogenic response in the periosteum for bone repair during socket healing. 
Choukroun et al ${ }^{18}$ used the PRF as a filling material in extraction socket; clinically they confirm the neovascularization and epithelial coverage of the extraction socket. PRF contains platelets and plasma rich in growth factors, Alpha granules of platelets include copious amount of growth factors such as platelet-derived growth factor (PDGF), platelet-derived endothelial growth factor (PDEGF), platelet-derived angiogenesis factor (PDAF), transforming growth factor beta (TGF-b), and epidermal growth factor (EGF) liberated from PRF network; these factors play an essential role fibroblasts recruitment, tissue reconstitution, with increased angiogenesis and re-establishing the vascular integrity, and finally covering of the wound. These factors enhance granulation tissue production and epithelialization and osteogenesis.

Within the clot in the socket, the osteogenic potential cells, invade the clot and lay down bone. In dry socket, where the clot has been disintegrated, wound healing is delayed, So Healing is benefited by the PRF by the development of microvascularization and cell migration into a wound, and hence fibrin network contains leukocytes and assists their movement that of great concern in infected wounds. Recent reports have suggested that more rapid epithelialization and greater bone regeneration, with a denser bone with mature well organized trabeculae occurs with PRF. ${ }^{16,20}$

Turner ${ }^{29}$ stated that alvogyl packing of the socket could delay socket healing and increase the risk of infection. Local irritant effect of eugenol and the delay in wound healing is well documented in the literature as reported by Sarrami et al ${ }^{30}$, Mainous ${ }^{31}$ and also by Navas and Mendoza ${ }^{32}$.

Statistically significant differences were observed in the pain scores among patients treated with PRF, Alvogyl and ZOE. Results showed that faster pain relief with Alvogyl, but also PRF was able to provide effective pain relief. Further, fewer visits to change dressing was required with PRF as compared to the other two medicaments. The findings suggest a statistically significant difference in healing potential outcome between the three groups, with PRF clearly showing optimistic improvement in clinical signs. However, further studies with larger sample size and longer follow-up periods are necessary to draw decisive conclusions.

\section{CONCLUSION}

In our study, we found that PRF is an appropriate dressing material as compared to the standard alvogyl and ZOE dressing for the management of dry socket. While all the three tested medicaments showed affirmative outcomes. The results of this study showed that (PRF) has good pain control with quick epithelisation of the socket and better and faster clinical socket healing with reduction of inflammation and fewer visits for dressing change.

\section{REFERENCES}

1. Blum IR. Contemporary views on dry socket (alveolar osteitis): a clinical appraisal of standardization, aetiopathogenesis and management: a critical review. Int $\mathbf{J}$ Oral Maxillofac Surg; 31(3):309-17, 2002.

2. Crawford JY. Dry socket. Dent Cosmos. ; 38:929-993, 1896.

3. Cardoso C L, Rodrigues M V, Ferreira O, Garlet G P, and de Carvalho PP, : Clinical concepts of dry socket. J Oral Maxillofac Surg.; 68: 1922-32, 2010.

4. Parthasarathi K, Smith A. and Chandu A.: Factors affecting incidence of dry socket: a prospective community-based study. J Oral Maxillofac Surg. ; 69:1880-4, 2011.

5. Kolokythas A, Olech E, and Miloro M.: Alveolar osteitis. : A comprehensive review of concepts and controversies. Int J Dent; 10:1-10, 2010.

6. Mamoun J.; Dry Socket Etiology, Diagnosis, and Clinical Treatment Techniques.J Korean Assoc Oral Maxillofac Surg.; 44:52-58. 2018.

7. Bowe DC, Rogers S, Stassen Leo FA. : The management of dry socket/alveolar osteitis. J Dent Assoc.; 57:305-10, 2011.

8. Garcia AG, Grana PM, Sampedro FG, Diago MP, Rey JM. Does oral contraceptive use affect the incidence of complications after extraction of a mandibular third molar? Br Dent J; 194:453-5, 2003. 
9. Oginno FO. Dry socket: a prospective study of prevalent risk factors in a Nigerian population. J Oral Maxillofac Surg.; 66:2290-2295, 2008.

10. Tarakji B, Saleh LA, Umair A, Azzeghaiby SN, Hanouneh S. Systemic review of dry socket: aetiology, treatment and prevention. J Clin Diagn Res.;9:10,2015.

11. Fernandes GJ, Hatton MN. Prevention of alveolar osteitis. A case report and review of literature. N Y State Dent $\mathrm{J}$ :;82:21-25,2016.

12. Wates E, Ria B. The prevention and management of dry socket. Do antibiotics have a role to play? Prim Dent $\mathrm{J} . ; 4: 42-43,2015$.

13. Sayed AR, Ahmed TE, Amira Z, Ali Fahd. Efficacy of Autologous Leucocyte-Platelet-rich Fibrin (L-PRF) versus Hydroxyapatite as a Graft Material for Socket Healing after Surgical Extraction of Impacted Mandibular Third Molars: A Comparative Clinical and Radiographic study. EDJ; 65, 89-100, 2019.

14. Khiyavi R, Barghi V, Yazdani J, et al. Effect of Biotene mouth wash on prevention of alveolar Osteitis after extraction of permanent Mandibular first molar Teeth. Elixir Hum Physiol. 2012;47:8672-8674

15. Jesudasan JS, Wahab PU, Sekhar MR. Effectiveness of $0.2 \%$ chlorhexidine gel and eugenol based paste on postoperative alveolar osteitis in patients having third molars extracted: a randomized controlled clinical trial. $\mathrm{Br}$ J Oral Maxillofac Surg.; 53:826-830, 2015.

16. Haraji A, Lassemi E, Motamedi M, Alavi M, Adibnejad S. Effect of plasma rich in growth factors on alveolar osteitis. Natl J Maxillofac Surg.; 3:38-41, 2012.

17. Pal US, Singh BP, Verma V. Comparative evaluation of zinc oxide eugenol versus gelatin sponge soaked in plasma rich in growth factor in the treatment of dry socket: an initial study. Contemp Clin Dent.; 4:37-41, 2013.

18. Choukroun J, Diss A, Simonpieri A, Girard MO, Schoeffler C, Dohan SL et al. Platelet-rich fibrin (PRF): a secondgeneration platelet concentrate. Part IV: clinical effects on tissue healing. Oral Surg Oral Med Oral Pathol Oral Radiol Endod; 101:56-60, 2006.

19. Dohan DM, Choukroun J, Diss A, Dohan SL, Dohan AJ, Mouhyi $\mathrm{J}$ et al. Platelet-rich fibrin (PRF): a second generation platelet concentrate, Part I: technological concepts and evolution. Oral Surg Oral Med Oral Pathol Oral Radiol Endod; 101:37-44, 2006.

20. Dohan DM, Choukroun J, Diss A, Dohan SL, Dohan
AJ, Mouhyi J et al. Platelet-rich fibrin (PRF): a secondgeneration platelet concentrate. Part II: platelet related biologic features. Oral Surg Oral Med Oral Pathol Oral Radiol Endod; 101:45-50. 2006

21. Dohan DM, Choukroun J, Diss A, Dohan SL, Dohan AJ, Mouhyi J et al. Platelet-rich fibrin (PRF): a second generation platelet concentrate. Part III: leucocyte activation: a new feature for platelet concentrates. Oral Surg Oral Med Oral Pathol Oral Radiol Endod;101:51-5,2006

22. Eshghpour M, Dastmalchi P, Nekooei AH, Nejat A. Effect of platelet-rich fibrin on frequency of alveolar osteitis following mandibular third molar surgery: a doubleblinded randomized clinical trial. J Oral Maxillofac Surg.; 72:1463-67, 2014.

23. Landry RG, Turnbull RS, Howley T. Effectiveness of benzydamine $\mathrm{HCl}$ in the treatment of periodontal postsurgical patients. Res Clin Forums, 10:105-18, 1988.

24. Ogata Y, and Hur Y.: A higher incidence of dry socket may be related to the use of oral contraceptives after impacted mandibular third-molar extraction. J Am Dent Assoc.; 147:840-2, 2016.

25. Almeida LE, Pierce S, Klar K, Sherman K: Effects of oral contraceptives on the prevalence of alveolar osteitis after mandibular third molar surgery: a retrospective study..Int J Oral Maxillofac Surg.; 45:1299-302, 2016.

26. Nusair YM, Younis MH: Prevalence, clinical picture, and risk factors of dry socket in a Jordanian dental teaching center. J Contemp Dent Pract.;8(3):53-63. 2007.

27. Akinbami BO. and Godspower T.: Dry socket: incidence, clinical features, and predisposing factors. Int $\mathrm{J}$ Dent. 10:1155, 2014.

28. Mudali V and, Mahomed O: Incidence and predisposing factors for dry socket following extraction of permanent teeth at a regional hospital in Kwa-Zulu Natal saDJ; 71: 166-169, 2016.

29. Turner PS. A clinical study of "dry socket". Int J Oral Surg; 11:226-31, 1982.

30. Sarrami N, Pemberton MN, Thornhill MH, Theaker ED. Adverse reactions associated with the use of eugenol in dentistry. Br Dent J; 193:257-9, 2002.

31. Mainous EG. Foreign body reaction after zinc oxide eugenol packing in localized osteitis. J Oral Surg; 32:207$8,1974$.

32. Navas RMA, Mendoza MGM. Case report: late complication of a dry socket treatment. Int J Dent, 2010:4. doi:10.1155/2010/479306 